\title{
Clinicopathological analysis of 156 patients with angiomyolipoma originating from different organs
}

\author{
LIN YANG $^{1 *}$, XIAO-LI FENG ${ }^{1 *}$, STEVEN SHEN $^{2}$, LING SHAN $^{1}$, \\ HAI-FENG ZHANG ${ }^{1}$, XIU-YUN LIU ${ }^{1}$ and NING LV ${ }^{1}$ \\ ${ }^{1}$ Department of Pathology, Cancer Hospital (Institute), Chinese Academy of Medical Sciences, \\ Peking Union Medical College, Beijing 100021, P.R. China; ${ }^{2}$ Pathology and Laboratory Medicine, \\ Methodist Hospital Research Institute, Weill Cornell Medical College, Cornell University, New York, NY, USA
}

Received September 26, 2011; Accepted December 14, 2011

DOI: $10.3892 / \mathrm{ol} .2012 .554$

\begin{abstract}
Angiomyolipoma (AML) is a benign mesenchymal tumor composed of variable proportions of adipose tissue, spindle and epithelioid smooth muscle cells and abnormal thick-walled blood vessels. Approximately one-third of epithelioid AML (EAML) cases have been reported to have metastasis. Thus, it would be of interest to identify the adverse pathological parameters correlated with outcome. However, few studies have been conducted on large numbers of samples. The aim of this study was to highlight the clinicopathological features of AML and the morphological features of EAML, which were correlated with malignant behaviors in patients from a single institutional series analysis. One hundred and fifty-six consecutive AMLs, correlating with pathological characteristics, were analyzed between 1981 and 2010. The Chi-square test was performed to clarify the significance of the clinicopathological factors among the regular and epithelioid subtypes with or without atypia. The two organs most commonly involved were the kidney $(77 \%, 120 / 156)$ and liver (14\%, 22/156). Of the 156 AMLs, EAMLs (17.3\%, 27/156) had more marked mitosis, hemorrhage and multinucleated giant cells compared with the regular AMLs $(82.7 \%, 129 / 156)$. The 11 EAMLs with atypia $(40.7 \%, 11 / 27)$ had more nucleoli and mitotic cells compared with the 16 EAMLs without atypia $(59.3 \%, 16 / 27 ; \mathrm{p}<0.05)$. Follow-up results of $79 \%$ of cases $(124 / 156)$ were obtained, and of these, only one kidney AML $(0.8 \%, 1 / 124)$ presented with liver metastasis one year after nephrectomy. This sizeable single institutional AML series
\end{abstract}

Correspondence to: Dr Ning Lv, Department of Pathology, Cancer Hospital (Institute), Chinese Academy of Medical Sciences, Peking Union Medical College, 17 South Panjiayuan Lane, Beijing 100021, P.R. China

E-mail:nlu03@126.com

*Contributed equally

Key words: angiomyolipoma, epithelioid angiomyolipoma, clinicopathological analysis, kidney, liver analysis revealed that the kidney and liver were the two most commonly involved organs, and most of the cases presented a benign clinical course. Few EAMLs were malignant, although adverse features including atypical mitotic figures, blood vessel invasion and tumor embolus may be significant in predicting malignant behavior.

\section{Introduction}

Angiomyolipoma (AML) is a relatively rare neoplasm, composed of variable proportions of adipose tissue, spindle and epithelioid smooth muscle-like cells and abnormal thick-walled blood vessels (1). Most AMLs are regarded as benign in clinical course, although certain cases have been reported to be recurrent or metastatic. Approximately $30 \%$ of epithelioid AMLs (EAMLs), which are partially or entirely composed of large epithelioid cells with abundant cytoplasm, vesicular nuclei and prominent nucleoli, have malignant potential (2). By contrast, an epithelioid morphology of the smooth muscle-like cells has been described in regular AMLs that are not associated with aggressive clinical behavior. This is analogous to the conventional description of smooth muscle neoplasm, in which epithelioid and conventional spindle cell leiomyomas exist; when referring to smooth muscle neoplasms, 'epithelioid' does not necessarily indicate atypia or increased risk of malignancy (2). Therefore, in 2010, Brimo et al proposed the concept of atypical EAMLs, and suggested that although not all EAMLs have malignant potential, certain atypical morphological features may be correlated with malignant behavior. These authors presented a predictive model of 4 atypical features, including: more than $70 \%$ atypical epithelioid cells; more than 2 mitotic figures per $10 \mathrm{HPF}$; atypical mitotic figures; and necrosis, which accurately categorized $78 \%$ of clinically malignant and $100 \%$ of clinically benign EAMLs with atypia (2). This proposal was established following an analysis of 40 cases of EAML with atypia. Whether it may be applied in general to predict malignant behavior requires further investigation. In this study, we analyzed 156 AMLs originating from different organs, which were classified into two groups (regular and epithelioid), and investigated whether the above proposal was suitable for distinguishing the cases with poor prognosis. 
Table I. Clinicopathological characteristics of 156 angiomyolipomas.

\begin{tabular}{|c|c|c|c|}
\hline $\begin{array}{l}\text { Clinicopathological } \\
\text { characteristics }\end{array}$ & $\begin{array}{l}\text { Kidney and perinephric/ } \\
\text { retroperitoneal }(n=122)\end{array}$ & $\begin{array}{l}\text { Liver } \\
(\mathrm{n}=22)\end{array}$ & $\begin{array}{l}\text { Other sites } \\
(\mathrm{n}=12)\end{array}$ \\
\hline \multicolumn{4}{|l|}{ Gender } \\
\hline Male & 40 & 6 & 7 \\
\hline Female & 82 & 16 & 5 \\
\hline \multicolumn{4}{|l|}{ Age } \\
\hline$\leq 40$ yrs & 39 & 8 & 6 \\
\hline $40-60$ yrs & 67 & 14 & 6 \\
\hline$>60$ yrs & 16 & 0 & 1 \\
\hline Average age (years) & 46 & 41 & 41 \\
\hline \multicolumn{4}{|l|}{ Maximum diameter $(\mathrm{cm})$} \\
\hline$\leq 5$ & 64 & 10 & 3 \\
\hline $5-10$ & 30 & 8 & 3 \\
\hline$>10$ & 21 & 4 & 3 \\
\hline Not known & 7 & 0 & 3 \\
\hline \multicolumn{4}{|l|}{ Histological features } \\
\hline Regular & 95 & 21 & 12 \\
\hline Epithelioid, no atypia & 17 & 0 & 0 \\
\hline Epithelioid, with atypia & 10 & 1 & 0 \\
\hline
\end{tabular}

In addition, although AMLs are mostly observed in the kidney and liver, other involved sites are sporadically reported in the hard palate (3), adrenal gland (4), anterior mediastinum (5), pancreas (6) and pelvis (7). Whether AMLs occurring in sites other than the kidney and liver have the same clinicopathological characteristics and prognosis was also investigated.

The aims of this study were to report the affected organs and histological and biological behavior of a large series of 156 consecutive AMLs in different sites and to assess whether EAMLs with atypia may be stratified prognostically based on clinicopathological features.

\section{Materials and methods}

Cases of AML. Surgical pathology files dating from between 1981 and 2010 at the Department of Pathology, Cancer Hospital, Chinese Academy of Medical Sciences, Beijing, China, were searched for AML. One hundred and fifty-six resection cases were found. Pathological slides were reviewed in all cases. The diagnosis of AML was made by two pathologists (X.-L.F. and L.Y.) separately. In the cases when the two pathologists disagreed, immunohisto-hemistry results were considered to reach a final diagnosis.

Immunohistochemistry. Immunohistochemical stains for melanocytic markers (including HMB-45 and Melan-A), pan-cytokeratins (clones AE1/AE3 and Cam 5.2), SMA and S-100 and other differential markers, including hepatocyte antigen (Hep), were performed when necessary. The antibodies and the standard streptavidin-biotin system were purchased from Zhongshan Goldenbridge Biotechnology Co., Ltd. (Beijing, China). The immunostaining pattern and results were assessed by X.-L.F. and L.Y. Adequate immunoreactive tissue samples were used as positive controls for each antibody. Negative controls were produced by omission of the primary antibody.

Classification of AMLs. In this investigation, AMLs were classified as regular, epithelioid with atypia and epithelioid without atypia according to Brimo et al (2). Regular AMLs were diagnosed when all 3 components were readily identified (each component accounting for $>10 \%$ of the tumor) and no epithelioid components were found. EAMLs were diagnosed when the epithelioid component accounted for $>10 \%$ of the tumor. Epithelioid with atypia in this investigation was restricted to morphological variants of cells with an epithelioid, as opposed to a spindle cell, pattern, with abundant cytoplasm and cytological atypia, which included atypical polygonal cells, vesicular nuclei, prominent nucleoli and nuclear size that exceeded twice the size of adjacent nuclei, with moderate to severe nuclear atypia (2). 'Epithelioid without atypia' refers to abundant cytoplasm without nuclear atypia or mitosis.

Pathological parameters. Tumor size was recorded according to the largest tumor diameter in macroscopy. The percentage of the epithelioid component, nucleoli, mitosis and microscopic lymph vascular invasion was recorded. The following pathologic parameters were also evaluated: coagulative tumor necrosis, hemorrhage, multinucleated giant cells and perinephric fat invasion.

Follow-up and statistical analysis. Clinical follow-up was achieved by return visit records or telephone call. A tumor was considered malignant when there was evidence of distant metastases or local recurrence following resection. The 
Table II. Twelve regular AMLs from sites other than renal or liver: Follow-up results.

\begin{tabular}{rrrlrcc}
\hline No. & Gender & Age & \multicolumn{1}{c}{ Involved sites } & Largest diameter $(\mathrm{cm})$ & Follow-up period (years) & Follow-up results \\
\hline 1 & $\mathrm{~F}$ & 28 & Small intestine & 6 & 3 & $\mathrm{NF}^{\mathrm{a}}$ \\
2 & $\mathrm{M}$ & 40 & Chest wall & 15 & 10 & $\mathrm{NF}$ \\
3 & $\mathrm{M}$ & 41 & Auricle of right ear & $\mathrm{NK}$ & $\mathrm{NK}$ & $\mathrm{NF}$ \\
4 & $\mathrm{M}$ & 38 & Right lower extremity & $\mathrm{NK}$ & $\mathrm{NK}$ & $\mathrm{NF}$ \\
5 & $\mathrm{~F}$ & 24 & Left forearm & 4 & 4 & $\mathrm{NF}$ \\
6 & $\mathrm{M}$ & 9 & Left anterior mediastinum & 11 & 2.5 & $\mathrm{NF}$ \\
7 & $\mathrm{M}$ & 72 & Left retromolar triangle & 4 & 3 & $\mathrm{NF}$ \\
8 & $\mathrm{~F}$ & 45 & Pelvic cavity & 10 & 15 & $\mathrm{NF}$ \\
9 & $\mathrm{~F}$ & 60 & Breast & 4 & 6 & $\mathrm{NF}$ \\
10 & $\mathrm{~F}$ & 33 & Peritoneal cavity & 20 & 8 & $\mathrm{NF}$ \\
11 & $\mathrm{M}$ & 48 & Left leg & $\mathrm{NK}$ & $\mathrm{NK}$ & $\mathrm{NF}$ \\
12 & $\mathrm{M}$ & 48 & Left thoracic cavity & 8 & 3.5 & $\mathrm{NF}$ \\
\hline
\end{tabular}

NF, no recurrence or metastasis was found; NK, not known; AML, angiomyolipoma; F, female; M, male.

Table III. Comparison of pathological features: Regular vs. epithelioid-type angiomyolipoma.

\begin{tabular}{lcc}
\hline $\begin{array}{l}\text { Clinicopathological } \\
\text { characteristics }\end{array}$ & $\begin{array}{c}\text { Regular } \\
(\mathrm{n}=129 ; \%)\end{array}$ & $\begin{array}{c}\text { Epithelioid } \\
(\mathrm{n}=27 ; \%)\end{array}$ \\
\hline $\begin{array}{l}\text { Location } \\
\text { Renal and perinephric }{ }^{\mathrm{a}}\end{array}$ & $96(74.4)$ & $26(96.3)$ \\
Liver & 21 & 1 \\
Other sites & 12 & 0 \\
Male:female ratio & $1.9: 1.0$ & $2.4: 1.0$ \\
Average diameter of tumor (cm) & 7 & 9 \\
Average age at presentation (years) & 45 & 42 \\
Symptoms at presentation & & \\
Pain & 32 & 9 \\
Mass & 6 & 3 \\
Incidental & 56 & 11 \\
Malaise & 21 & 2 \\
Other symptoms $_{\text {Necrosis }}{ }^{\mathrm{a}}$ & 14 & 2 \\
Mitosis $^{\mathrm{a}}$ & $4(3.1)$ & $4(14.8)$ \\
Hemorrhage $^{\text {a }}$ & 0 & $10(37.4)$ \\
Multinucleated giant cells $^{\mathrm{a}}$ & $3(2.3)$ & $8(29.6)$ \\
Infiltrating boundary & 0 & $5(18.5)$ \\
\end{tabular}

${ }^{\mathrm{a}}$ Features that presented statistical significance between the regular and epithelioid subtypes.

above-mentioned clinicopathological factors were correlated with follow-up results. The Chi-square test was performed to clarify the various significances of the above factors among the regular and epithelioid subtypes with or without atypia. $\mathrm{P}<0.05$ was considered to indicate a statistically significant result.

\section{Results}

Involvement of AML in various sites. The clinicopathological characteristics of the 156 AMLs are shown in Table I. The kidney and liver were the most commonly involved organs, accounting for approximately $92 \%$ of the AMLs. Other rarely involved sites and the follow-up results are shown in Table II. Of the patients with involvement of the kidney, the mean age was 46 years and the female:male ratio was 2:1. In the patients with involvement of the liver, these values were 41 years and 2.5:1, respectively. These results revealed a female and mid-age (41-60 years) morbidity predominance in both the kidney and liver cases. The average largest diameter of the tumors was $6.6 \mathrm{~cm}$ (range, $1-35 \mathrm{~cm}$ ). Six of the kidney cases presented with multiple foci. Two of the cases had foci located in the bilateral kidney and the remaining four cases had multiple foci in the unilateral kidney. Of the six cases with multiple foci, three were available for a follow-up, the results of which revealed no increase in size over a period of 1-3 years.

Of the 156 AML cases, 27 were EAMLs, of which 26 were located in the kidney and one in the liver. Morphologically, the regular AMLs had three components in different proportions, including abnormal blood vessels, smooth muscle-like cells and adipose tissue. The percentage of adipose tissue, smooth muscle and epithelioid components was recorded for all AMLs in this study. We found that certain tumors consisted almost exclusively $(>80 \%)$ of one component, for example adipose tissue ( 5 cases) or smooth muscle ( 2 cases). These were termed monophasic types. Of the 7 monophasic AMLs, the largest diameter ranged from 3 to $29 \mathrm{~cm}$ and the mean age of the patients was 46 years. None of the monophasic AMLs were recurrent or metastatic within the duration of the follow-up (12-96 months).

Correlation of AMLs and pathological parameters. The AMLs included in this investigation were classified into two types according to cell morphological features: regular and epithelioid. The pathological parameters were compared, 
Table IV. Clinicopathological characteristics of epithelioid AMLs with or without atypia.

\begin{tabular}{|c|c|c|}
\hline $\begin{array}{l}\text { Clinicopathological } \\
\text { characteristics }\end{array}$ & $\begin{array}{c}\text { EAML, no atypia } \\
(\mathrm{n}=16 ; \%)\end{array}$ & $\begin{array}{c}\text { EAML, with atypia } \\
(\mathrm{n}=11 ; \%)\end{array}$ \\
\hline \multicolumn{3}{|l|}{ Clinical features } \\
\hline \multicolumn{3}{|l|}{ Location } \\
\hline Kidney & 16 & 10 \\
\hline Liver & 0 & 1 \\
\hline Male:female ratio & $3: 5$ & $2: 9$ \\
\hline Average diameter of tumor $(\mathrm{cm})$ & 9 & 11 \\
\hline Average age at presentation (years) & 41 & 42 \\
\hline \multicolumn{3}{|l|}{ Symptoms at presentation } \\
\hline Pain & 7 & 3 \\
\hline Mass & 0 & 1 \\
\hline Incidental & 8 & 4 \\
\hline Malaise & 1 & 2 \\
\hline Other symptoms & 0 & 1 \\
\hline \multicolumn{3}{|l|}{ Pathological features } \\
\hline Envelope & $5(31.3)$ & $3(27.3)$ \\
\hline Epithelioid component $>70$ & $2(12.5)$ & $5(45.5)$ \\
\hline Nucleoli & $1(6.3)$ & $8(72.7)^{\mathrm{a}}$ \\
\hline Mitosis & $4(25.0)$ & $9(81.8)^{\mathrm{a}}$ \\
\hline Multinucleated giant cells & $3(18.8)$ & $5(45.5)$ \\
\hline Necrosis & $2(12.5)$ & $5(45.5)$ \\
\hline Hemorrhage & $5(31.3)$ & $7(63.6)$ \\
\hline Tumor embolus & 0 & $2(18.2)$ \\
\hline Infiltrating boundary & $2(12.5)$ & $4(36.4)$ \\
\hline \multicolumn{3}{|l|}{ Follow-up } \\
\hline Case no. () & $12(75)$ & $8(80)$ \\
\hline Mean follow up duration (months) & 84 & 50 \\
\hline Recurrence or metastasis & None & 1 liver metastasis \\
\hline
\end{tabular}

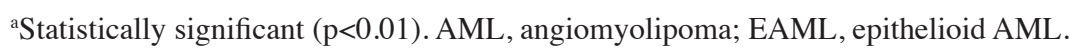

including necrosis, hemorrhage, mitosis, multinucleated giant cells and infiltration of perinephric fat (Table III). This comparison revealed that epithelioid AMLs have more marked mitosis, hemorrhage and multinucleated giant cells than regular AMLs. EAMLs were also subdivided into two categories according to Brimo et al (2): EAML without atypia and EAML with atypia. Nucleoli and mitosis were significantly more in EAML with atypia than in EAML without atypia $(\mathrm{p}<0.05)$.

Follow-up. The follow-up information from 124 out of the 156 AMLs was obtained. None of the cases presented with recurrence, except one case of renal EAML with atypia, which exhibited liver metastasis one year after nephrectomy. This patient was a 25-year-old female who carried a mass $15 \mathrm{~cm}$ in diameter in the right kidney and a swollen lymph node $5 \mathrm{~cm}$ in diameter between the abdominal aorta and inferior vena cava at presentation. Microscopically, the epithelioid component in this case accounted for $90 \%$ of the tumor, which was consistent with the diagnosis of EAML. Atypical features were clearly observed, including massive necrosis, nucleated or multinucleated giant cells, multiple acidophilic nucleoli, increased ratio of nucleus to cytoplasm, 4/10 HPF mitosis, multiple foci of blood vessels and perinephric fat invasion. Follow-up revealed a solid mass of size $3.5 \times 3.5 \times 2.0 \mathrm{~cm}$ in the liver 12 months later. The morphology of the liver tumor was similar to that of the kidney and a tumor embolus was observed in the liver parenchyma around the tumor. Immunohistochemical staining of the liver lesion presented HMB45 (2+), actin (3+), SMA (3+), S-100 (1+, patchy) and CD34 (-), whereas CK18, CK8, AE1/ AE3 and Hep were negative. The final diagnosis was metastatic epithelioid angiomyolipoma. 


\section{Discussion}

In this single institutional investigation, we studied 156 AMLs originating from different organs and compared the follow-up results with clinicopathological characteristics. We found 12 AMLs that originated from a location other than the kidney or liver, which presented as a classically triphasic phenotype, had a benign clinical course and no observed epithelioid components. One hundred and twenty tumors occurred in the kidney $(77 \%, 120 / 156)$. Few systematic large series analyses were found in the literature, except for those on renal AMLs focused on populations in Korea and the USA $(8,9)$. Kyo et al reported 129 renal cases in Korea, of which only one female patient experienced recurrence (9). Another 194 American cases were presented by Aydin et al, of which none had local recurrence or distant metastasis during a mean followup time of 5.1 years (8). Our data identified 26 EAMLs out of 120 renal AMLs. Only one case presented lymph node and liver metastasis 12 months after nephrectomy and no AML-related mortality occurred during a mean follow-up period of 7.0 years, which was consistent with the abovementioned studies on populations in Korea and the USA. It is suggested that AMLs have almost the same low incidence rate in populations in the USA, Korea and China.

EAML is a variant of AML and, according to the WHO guidelines, approximately one-third of EAMLs have potential malignant behavior, exhibiting local recurrence or distant metastasis (1). A number of published case studies and small anecdotal series revealed a controversial prognosis of EAML (10-15). There was no conformity among different investigators concerning the criteria for malignancy and worse prognosis parameters in EAMLs. The generally affirmative malignant criteria included cell polymorphism, atypical features, mitosis, hemorrhage, necrosis, blood vessel and lymph node invasion and local recurrence. Other investigators considered metastasis as the only acceptable criterion in the diagnosis of a malignant AML (16). In a study of 40 cases of EAML, Brimo et al proposed four pathological features, which are predictive of malignancy in epithelioid AML with atypia. These features were: i) $\geq 70 \%$ atypical epithelioid cells; ii) $\geq 2$ mitotic figures per $10 \mathrm{HPF}$; iii) atypical mitotic figures; iv) necrosis. Additionally, these authors concluded that the presence of three or more of the above features predicts an increased risk of clinically malignant behavior. This model accurately classified $78 \%$ of the clinically malignant and $100 \%$ of the clinically benign epithelioid AMLs with atypia (2).

In this investigation, we identified 11 atypical EAML cases according to the proposal by Brimo et al (Table IV). Only one of the 11 atypical cases presented with metastasis. This case presented 6 atypical features including blood vessel invasion and lymph node metastasis plus the above four adverse features, as discussed by Brimo et al (2). The other 10 cases had no recurrence or metastasis in a mean follow-up period of 42 months (range, 9-60 months), which was not consis- tent with the results of Brimo et al. The authors suggest that blood vessel invasion or tumor embolus is as significant as other morphological features in diagnosing atypical EAMLs. However, there were only 11 atypical EAML cases in this investigation. More cases are required in order to draw more precise conclusions.

In conclusion, AMLs mostly occur in the kidney and the liver, and most present a benign clinical course. EAMLs were few in our investigation. Adverse features, including atypical mitotic figures, blood vessel invasion and tumor embolus, may be significant in predicting malignant behavior.

\section{References}

1. Eble JN, Sauter G, Epstein JI, et al (eds): World Health Organization Classification of Tumors. Pathology and Genetics of Tumors of the Urinary System and Male Genital Organs. IARC Press, Lyon, 2004.

2. Brimo F, Robinson B, Guo C, et al: Renal epithelioid angiomyolipoma with atypia: a series of 40 cases with emphasis on clinicopathologic prognostic indicators of malignancy. Am J Surg Pathol 34: 715-722, 2010.

3. Alvarez AC, Fernández SJ, Fernández CM, et al: Sporadic oral angiomyolipoma. Case report. Med Oral Patol Oral Cir Bucal 12: E391-393, 2007.

4. Elsayes KM, Narra VR, Lewis JS Jr, et al: Magnetic resonance imaging of adrenal angiomyolipoma. J Comput Assist Tomogr 29: 80-82, 2005.

5. Amir AM, Zeebregts CJ and Mulder HJ: Anterior mediastinal presentation of a giant angiomyolipoma. Ann Thorac Surg 78: 2161-2163, 2004.

6. Heywood G, Smyrk TC and Donohue JH: Primary angiomyolipoma of the pancreas. Pancreas 28: 443-445, 2004.

7. Gronchi A, Diment J, Colecchia M, et al: Atypical pleomorphic epithelioid angiomyolipoma localized to the pelvis: a case report and review of the literature. Histopathology 44: 292-295, 2004.

8. Aydin H, Magi-Galluzzi C, Lane BR, et al: Renal angiomyolipoma clinicopathologic study of 194 cases with emphasis on the epithelioid histology and tuberous sclerosis association. Am J Surg Pathol 33: 289-297, 2009.

9. Kyo CK, Won TK, Won SH, et al: Trends of presentation and clinical outcome of treated renal angiomyolipoma. Yonsei Med J 51: 728-734, 2010.

10. Kato I, Inayama Y and Yamanaka S: Epithelioid angiomyolipoma of the kidney. Pathol Int 59: 38-43, 2009.

11. Pea M, Bonetti F, Martignoni G, et al: Apparent renal cell carcinomas in tuberous sclerosis are heterogeneous: the identification of malignant epithelioid angiomyolipoma. Am J Surg Pathol 22: 180-187, 1998.

12. Faraji H, Nguyen BN and Mai KT: Renal epithelioid angiomyolipoma: a study of six cases and a meta-analytic study. Development of criteria for screening the entity with prognostic significance. Histopathology 55: 525-534, 2009.

13. Tsai CC, Wu WJ, Li CC, et al: Epithelioid angiomyolipoma of the kidney mimicking renal cell carcinoma: a clinicopathologic analysis of cases and literature review. Kaohsiung J Med Sci 25: 133-140, 2009.

14. Zhang HY, Qin X, Wang XJ, et al: 60 cases of renal angioleiomyolipoma: a clinicopathologic analysis. Tumor 23: 329-331, 2003 (In Chinese).

15. Nguyen TT, Gorman B, Shields D, et al: Malignant hepatic angiomyolipoma: report of a case and review of literature. Am J Surg Pathol 32: 793-798, 2008

16. Cho NH, Shim HS, Choi YD, et al: Estrogen receptor is significantly associated with the epithelioid variants of renal angiomyolipoma: A clinicopathological and immunohistochemical study of 67 cases. Pathol Int 54: 510-515, 2004. 